\title{
Interdisciplinary Aspects of Chronic Inflammatory Bowel Diseases
}

\author{
Georg Kähler $^{\mathrm{a}} \quad$ Ulrich Böcker $^{\mathrm{b}}$ Robert Ehehalt ${ }^{\mathrm{c}} \quad$ Martin Götz $^{\mathrm{d}}$ \\ Peter Hasselblatt ${ }^{\mathrm{e}}$ Martin E. Kreis ${ }^{\mathrm{f}}$ Anton J. Kroesen ${ }^{\mathrm{g}}$ \\ ${ }^{a}$ Central Interdisciplinary Endoscopy, Medical Center Mannheim, University of Heidelberg, Mannheim, Germany; \\ ${ }^{b}$ Klinik für Innere Medizin - Gastroenterologie, Diabetologie und Hepatologie, Vivantes Klinikum Neukölln, Berlin,

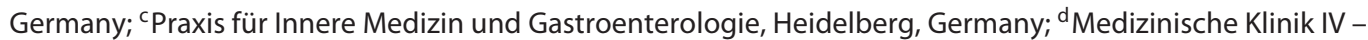 \\ Gastroenterologie/Onkologie, Klinikum Sindelfingen-Böblingen, Böblingen, Germany; ${ }^{e}$ Klinik für Innere Medizin \\ II - Gastroenterologie, Hepatologie, Endokrinologie und Infektiologie, Universitätsklinikum Freiburg, Freiburg, \\ Germany; ${ }^{f}$ Klinik für Allgemein-, Viszeral- und Gefäßchirurgie, Charité - Universitätsmedizin Berlin, Campus \\ Benjamin Franklin, Berlin, Germany; ${ }^{9}$ Allgemein-Viszeral- und Unfallchirurgie, Krankenhaus Porz am Rhein, Cologne, \\ Germany
}

\section{Question 1: What Is the Best Surveillance for Patients with Inflammatory Bowel Disease? Ultrasound, Endoscopy, Lab, or Just Clinical?}

Böcker: Best cancer surveillance is endoscopy, best stricture surveillance are clinical symptoms, best (distal) inflammatory surveillance is calprotectin in stools.

Ehehalt: Gold standard would be endoscopy; however, from a practical aspect, clinic together with lab is leading.

Götz: Cancer surveillance: endoscopy.

Response to therapy: clinical, lab: frequently - ultrasound: under evaluation, probably good (bowel wall thickness, Limberg score) - endoscopy: optimal, but invasive and reserved for special situations.

Hasselblatt: Best routine surveillance of inflammatory bowel disease patients is clinical in combination with calprotectin (should be $<250$ ). In case of symptoms, we add ultrasound, endoscopy where necessary (or indicated, e.g., for tumor surveillance and if the results affect therapy), and eventually MRI.

Kreis: All of each in an adequate frequency.

Kroesen: It depends on the individual situation of the patient. In general, ultrasound and clinical examination are enough. A sudden deterioration or increasing stenotic complaints can make an endoscopy necessary, especially when surgery or endoscopic dilatation becomes a more reasonable treatment option.

\section{Question 2: Anastomotic Strictures: How Often and with Which Diameter Do You Dilate or Do You Recommend Re-Anastomosis?}

Böcker: If limited extent of the stricture and patient consents, always trial of endoscopic treatment with fractionated balloon dilatation before surgery. Perform as many times as necessary until an average diameter of $15 \mathrm{~mm}$ is reached.

\section{KARGER}

(C) 2019 S. Karger AG, Basel
Prof. Dr. Georg Kähler

Central Interdisciplinary Endoscopy, Medical Center Mannheim

University of Heidelberg, Theodor-Kutzer-Ufer 1-3

DE-68167 Mannheim (Germany)

E-Mail georg.kaehler@umm.de 
Ehehalt: Maximum $3 \mathrm{~cm}$ length of stenosis, dilatation to max. $2 \mathrm{~cm}$.

Götz: 15 or $18 \mathrm{~mm}$ (depending on residual lumen width).

Repetition depends on initial response (IR): IR good: as often as necessary - IR intermediate: consider surgery - IR: none: surgery.

Hasselblatt: We usually dilate up to $20 \mathrm{~mm}$ (of course depending on the initial diameter). If inflammation is present, we add/increase anti-inflammatory therapy. Repeat exams are scheduled if symptoms persist/relapse. Otherwise, we try to do an endoscopic control (with dilatation) after 3-6 months to assure patency (and check for mucosal healing if possible). We do not perform scheduled repeated dilatations (as done for esophageal strictures).

Kreis: There is no absolute number. We make it depend on the duration of the success of dilatation. Diameter: usually $18 \mathrm{~mm}$.

Kroesen: The basic criteria for endoscopic dilatation must be fulfilled: length under $3 \mathrm{~cm}$, no fistula, no kinking orally to the stricture. The patient has to be consented about the advantages and disadvantages of endoscopic dilatation and surgical resection and his decision is very important. Patients who are not sure about the necessity of an operation can be psychologically prepared by a dilatation even when the effect does not last that long.

Question 3: How Many Surgical Inflammatory Bowel Disease Operations per Year Are Needed for a Center?

Böcker: 25.

Ehehalt: 10 pouches.

Götz: 20.

Hasselblatt: Would suggest $>50$ inflammatory bowel disease-related surgeries and $>10$ proctocolectomies. Surgery should be done by laparoscopy where possible.

Kreis: 300 abdominal operations.

Kroesen: For ulcerative colitis, two studies exist. Both say that at least 10 proctocolectomies with ileoanal pouch should be performed per year. This is the lowest number which is acceptable. In Crohn's disease, a comparable number but in accordance with each operation can be postulated too. But due to the broad variety in Crohn's disease, it is more difficult to name an exact number. By heart, I would postulate more than 50 major abdominal surgeries.

\section{Question 4: What Are the Criteria for a Primary Failure of Medical Treatment? Can Surgery Be Too Early?}

Böcker: Failure means that the selected treatment goal has not been reached in a medication-specific time interval. Criteria relate to the selected goal that, as part of the treat-to-target discussion before initiating treatment, has been chosen, e.g., clinical, endoscopic, or other. Surgery can be too early if the postoperative anatomical and functional status results in relevant patient morbidity and impairs his/her quality of life but could have been avoided by intensified medical treatment with a reasonable chance/risk calculation, e.g., resection of a long small bowel segment.

Ehehalt: I do not understand that question. Do you mean steroid failure? Biologic failure? Of course, a high fibrostenotic stenosis would be a criterion.

Götz: Clinical non-response.

Surgery can be both too early or too late.

Surgery too early: depends on extent of resection (e.g., proctocolectomy) - the greater the extent, the more reluctant I am (and are the patients) to undergo surgery.

Hasselblatt: Primary failure means that there is no clinical/endoscopic improvement within 8-10 weeks. Criteria depend on the initial symptoms. Surgery is not a failure of therapy but can be a very reasonable alternative to medical therapies if combined with postoperative prophylaxis. It should always be discussed if biologicals fail. Upon failure of conventional therapy, surgery may be a reasonable alternative to anti-TNF.

Kreis: Continued symptoms, high levels of leukocytes, $\mathrm{CRP}$ and calprotectin, inflammation on imaging (ultrasound, endoscopy, MRI).

Kroesen: Just in a patient who did not receive any medical treatment, surgery can be too early. As soon as the main indications are met, surgery is indicated. For the indication refractory course, continuous corticosteroid use plus biologicals or start of a third-line biological therapy should be the latest point in time for surgery. 


\section{Participants}

Prof. Dr. med. Ulrich Böcker

Vivantes Klinikum Neukölln

Klinik für Innere Medizin - Gastroenterologie, Diabetologie und Hepatologie

Rudower Strasse 48, DE-12351 Berlin (Germany)

E-Mail ulrich.boecker@vivantes.de

Prof. Dr. Robert Ehehalt

Praxis für Innere Medizin und Gastroenterologie

Burgstrasse 61, DE-69121 Heidelberg (Germany)

E-Mail re@hd-gastro.de

Prof. Dr. med. Martin Götz

Chefarzt der Med. Klinik IV - Gastroenterologie/Onkologie

Klinikum Sindelfingen-Böblingen

Bunsenstrasse 120, DE-71032 Böblingen (Germany)

E-Mailm.goetz@klinikverbund-suedwest.de
PD Dr. med. Peter Hasselblatt

Klinik für Innere Medizin II - Gastroenterologie, Hepatologie, Endokrinologie und Infektiologie

Universitätsklinikum Freiburg

Hugstetter Strasse 55, DE-79106 Freiburg (Germany)

E-Mail Peter.Hasselblatt@uniklinik-freiburg.de

Univ.-Prof. Dr. med. Martin E. Kreis

Klinik für Allgemein-, Viszeral- und Gefäßchirurgie

Charité Universitätsmedizin Berlin

Campus Benjamin Franklin

Hindenburgdamm 30, DE-12200 Berlin (Germany)

E-Mail martin.kreis@charite.de

Prof. Dr. Anton J. Kroesen

Allgemein-Viszeral- und Unfallchirurgie

Krankenhaus Porz am Rhein

Urbacher Weg 19, DE-51149 Köln (Germany)

E-Mail a.kroesen@khporz.de 